\title{
USO DE UN SOFTWARE DE EVALUACIÓN DEL APRENDIZAJE PARA MEJORAR LOS RESULTADOS ACADÉMICOS
}

\author{
Carlos Antonio Li Loo Kung, ${ }^{1 *}$ Susy Karina Dávila Panduro² \\ ${ }^{1}$ Facultad de Industrias Alimentarias, Universidad Nacional de la Amazonía Peruana, c/ Nauta $5^{\text {ta }}$ cuadra, Iquitos, Perú. \\ ${ }^{2}$ Facultad de Ciencias de la Educación y Humanidades, Universidad Nacional de la Amazonía Peruana, c/ Sgto. Lores 6 ta cuadra, Iquitos, \\ Perú. \\ *e-mail: carlosli22@hotmail.com
}

\section{RESUMEN}

La investigación tuvo como objetivo: Aplicar un software de evaluación del aprendizaje para mejorar los resultados académicos en estudiantes, de la Facultad de Industrias Alimentarias de la Universidad Nacional de la Amazonía Peruana, Iquitos en el año 2011. El estudio pertenece al tipo experimental y el diseño fue el pre-experimental de tipo pre-test y post-test con un solo grupo. La población estuvo conformada por los estudiantes de la Escuela de Ingeniería en Industrias Alimentarias, de la Universidad Nacional de la Amazonia Peruana que fueron 205, la determinación de la muestra fue en forma no probabilística intencional conformada por 62 estudiantes. La técnica que se empleó en la recolección de los datos fue: la encuesta. El instrumento fue el cuestionario. Para el análisis e interpretación de los datos se empleó la estadística descriptiva: frecuencia, promedios simples, porcentaje y la estadística inferencial no paramétrica Chi-cuadrado $\left(\mathrm{X}^{2}\right)$, obteniendo $X^{2}{ }_{c}=69,04 ; X^{2}{ }_{t}=9,21$; es decir como $X^{2}{ }_{c}>X^{2} t, g l=2, \propto=0,01$; aceptando la hipótesis de investigación: "El software de evaluación de los aprendizajes ayudará a mejorar la obtención de resultados académicos de estudiantes, en la Facultad de Industrias Alimentarias de la Universidad Nacional de la Amazonía Peruana, durante el año 2011".

Palabras clave: Software, evaluación, aprendizaje, resultados académicos.

\section{USAGE OF SOFTWARE OF LEARNING ASSESSMENT TO IMPROVE THE ACADEMIC RESULTS}

\begin{abstract}
The objective of this research is to: Apply software learning assessment in order to enhance the attainment of academic results of students, in the Faculty of Food Industry, of National University of the Peruvian Amazon, Iquitos - 2011. The current study is of an experimental type and the design was a pre-experimental, one type pre-test and post-test with a single group.The population was formed by students from the School of Food Engineering, Faculty of Food Industries, National University of the Peruvian total of 205, the selection of the sample was not randomized but intentional and consisted of 62 students. The technique used in collecting the data was: The survey and the instrumentwasthequestionnaire.For the data processing computerprogram SPSS spanishversion 17 was used was obtained with the data matrix that organized the information into tables and graphs. For the analysis and interpretation of data was descriptive statistics: frequency, simple averages and percentages and the inferentialstatisticsnonparametric Chisquare $\left(X^{2}\right)$.Fortheconfirmingthe main hypothesis I used the nonparametric inferential statistics test Chi-square $\left(X^{2}\right)$, with $\propto=0,01 ; \mathrm{gl}=2$ getting $X^{2}{ }_{c}=69,04 ; X^{2}{ }_{t}=9,21$; ie $X^{2}{ }_{c}>X^{2}{ }_{t}$, with $>X^{2}{ }_{t}$ is accepted the hypothesis of the research: "The learning assessment software in the will help improvement of the academic outcome of students in the Faculty of Food Industry, National University of the Peruvian Amazon in 2011."
\end{abstract}

Keywords: Software, Evaluation, learning, academic result. 


\section{INTRODUCCIÓN}

La tecnología y la microcomputadora nunca reemplazarán al maestro en sus papeles como persona que planea e imparte la enseñanza. Pero los maestros con gran intuición consideran que la computadora puede mejorar la planeación de una clase y la conducción de la enseñanza y por lo tanto estimular el proceso de enseñanza y aprendizaje (Eiser y Salpeter, 2001).

Es esencial que a los estudiantes se les proporcione los objetivos de desempeño como del proceso. Estos les ayudarán a saber lo que se requiere de ellos cuando trabajen en las microcomputadoras. Como una herramienta de enseñanza, la microcomputadora también se puede programar para procesar los resultados de aprendizaje del individuo y del grupo. Esta capacidad de procesamiento ahorra un tiempo invaluable de registro del maestro (Aguirre, 2000).

Las microcomputadoras también proporcionan un medio por el cual el ejercicio y la práctica - así como la enseñanza individualizada - se pueden hacer parte de la rutina del aula. Los objetivos de desempeño se deben construir en programas tales que los estudiantes comprendan qué grado de precisión se requiere en un sistema individualizado (Alvarez, 1997).

Las microcomputadoras fácilmente permiten a los estudiantes establecer su propio ritmo. Para esto se requiere que cada uno de los usuarios conozca los resultados y patrones que se esperan. Finalmente, los programas interactivos de computadora le permiten al estudiante lograr tanto los objetivos de desempeño como los de proceso con una rapidez y eficiencia que ningún otro sistema puede lograr (Hidalgo, 2001).

El Software de Registro y Evaluación ver 2.0 (SRE), es un amplio programa para proporcionar a los docentes, las herramientas tecnológicas para mejorar su rendimiento y calidad en los procesos de evaluación que se realizan en las universidades, pudiendo crear los exámenes, ejecutarlos en forma automática, obtener registros y realizar las operaciones básicas de manejo de notas (Norris, 2005).

El Software de Registro y Evaluación tiene dos accesos directos, el primero será: "Software de Registro y Evaluación", que permite realizar todas las operaciones del programa (Módulo docente); y el segundo es: "SRE - Módulo de Evaluación", que es el que permite que los estudiantes rindan su evaluación.

\section{MATERIALES Y MÉTODOS}

El estudio fue experimental, y el diseño pre-experimental de tipo pre-test y post-test con un solo grupo; porque se aplicó el tratamiento en un solo momento y se recogió la información antes y después de la aplicación; donde se puso a prueba el software de evaluación del aprendizaje, dentro de un grupo de estudiantes en una asignatura definida, se identificó la percepción de los estudiantes para la obtención de resultados académicos, en la Facultad de Industrias Alimentarias de la Universidad Nacional de la Amazonia Peruana (Orlich, 2006).

La población del presente estudio estuvo conformado por todos los estudiantes de la Escuela de Ingeniería en Industrias Alimentarias, de la Universidad Nacional de la Amazonia Peruana, que fueron 205.

La muestra fue no probabilística intencional y estuvo conformada por el $30 \%$ de la población, siendo un total de 62 estudiantes de la Escuela de Ingeniería en Industrias Alimentarias, de la Universidad Nacional de la Amazonia Peruana.

La técnica que se empleó teniendo en cuenta el tamaño de la muestra fue la encuesta y el instrumento que se empleó en la recolección de la información fue el cuestionario (Rosi, 2002). 


\section{RESULTADOS Y DISCUSIÓN}

Antes de la aplicación del software de evaluación, se tiene como dato resaltante que en mayor porcentaje los estudiantes opinaron que los resultados académicos, son deficientes en todos los indicadores (Figura 1).

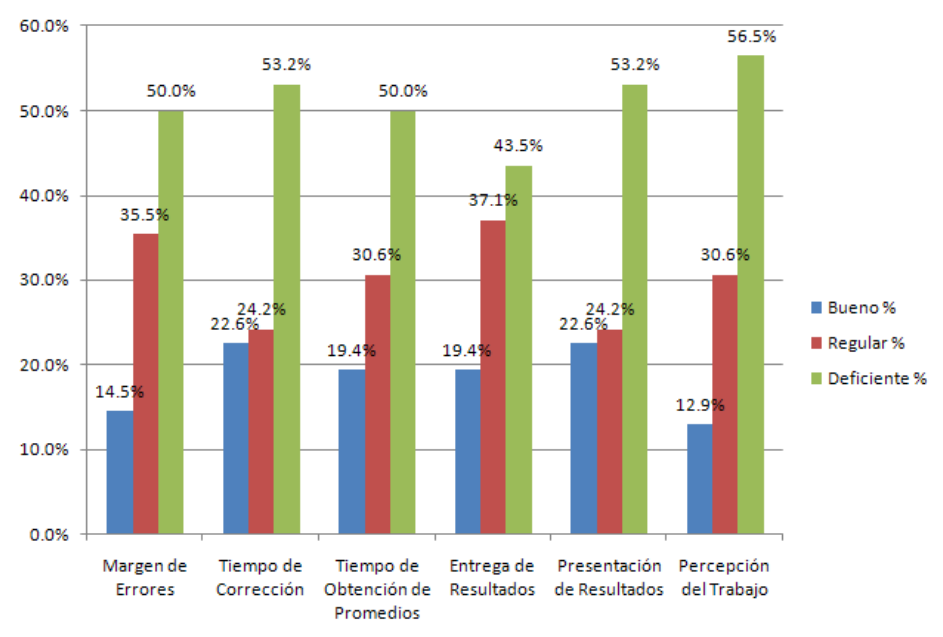

Figura 1. Opinión de los estudiantes acerca de los resultados académicos, antes de la aplicación del Software de Evaluación de Aprendizajes

Después de la aplicación del Software, se tiene como dato resaltante que en mayor porcentaje los estudiantes opinaron que los resultados académicos son buenos en todos los indicadores (Figura 2).

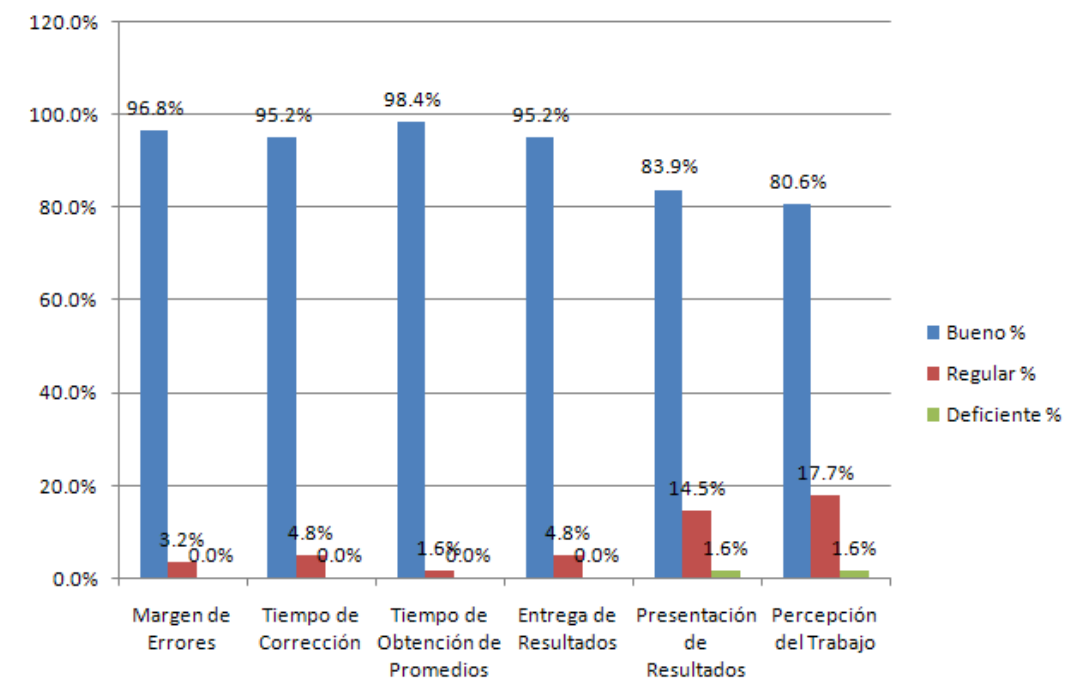

Figura 2: Opinión de los estudiantes acerca de los resultados académicos, después de la aplicación del Software de Evaluación de Aprendizajes.

En la Figura 3, se muestran los resultados de la opinión de los estudiantes sobre el uso de un software de evaluación, teniendo como dato resaltante que los estudiantes en su mayoría opinaron que es muy importante para mejorar el proceso de evaluación, pero consideran que los docentes, en su conjunto, no están capacitados para utilizarlo, así también indican que la infraestructura de la universidad no es la adecuada para su aplicación. 
Cienc. amaz., Vol. 2, No. 1, 2012

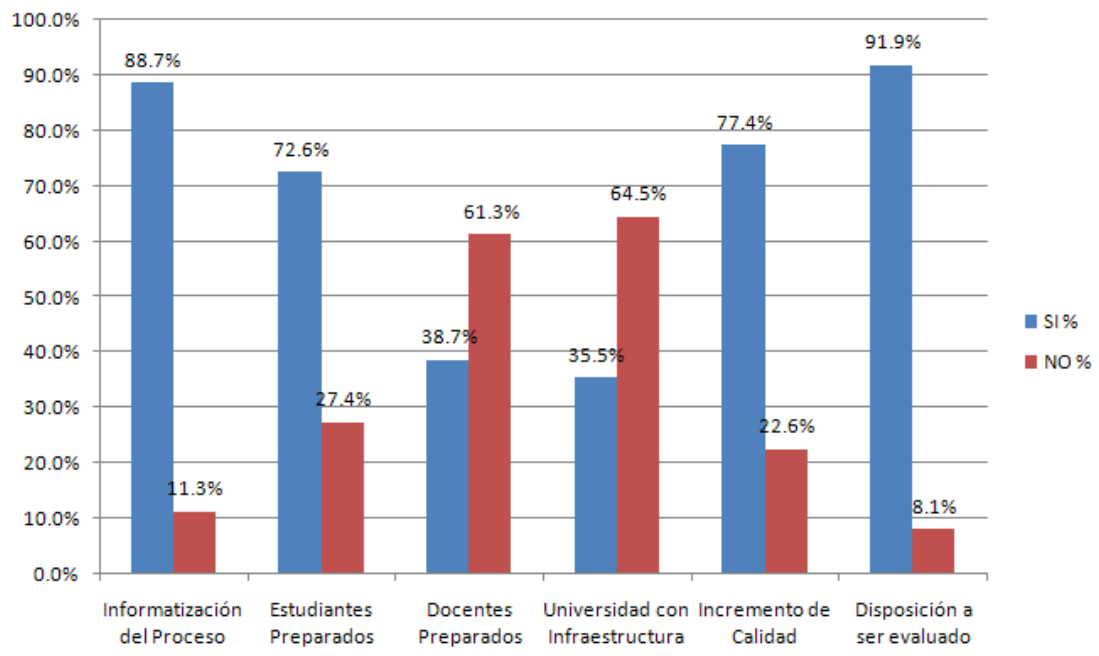

Figura 3: Opinión sobre la necesidad de un Software de Evaluación de los Aprendizajes para la Obtención de Resultados Académicos.

En la Figura 4, se tiene como dato resaltante que los estudiantes opinaron que el software de evaluación, es bueno para ser aplicado ytodas sus características como: facilidad de acceso, sistema de protección, confidencialidad, presentación, claridad, manejo de registro, adecuación, requerimientos y compatibilidad, son buenas.

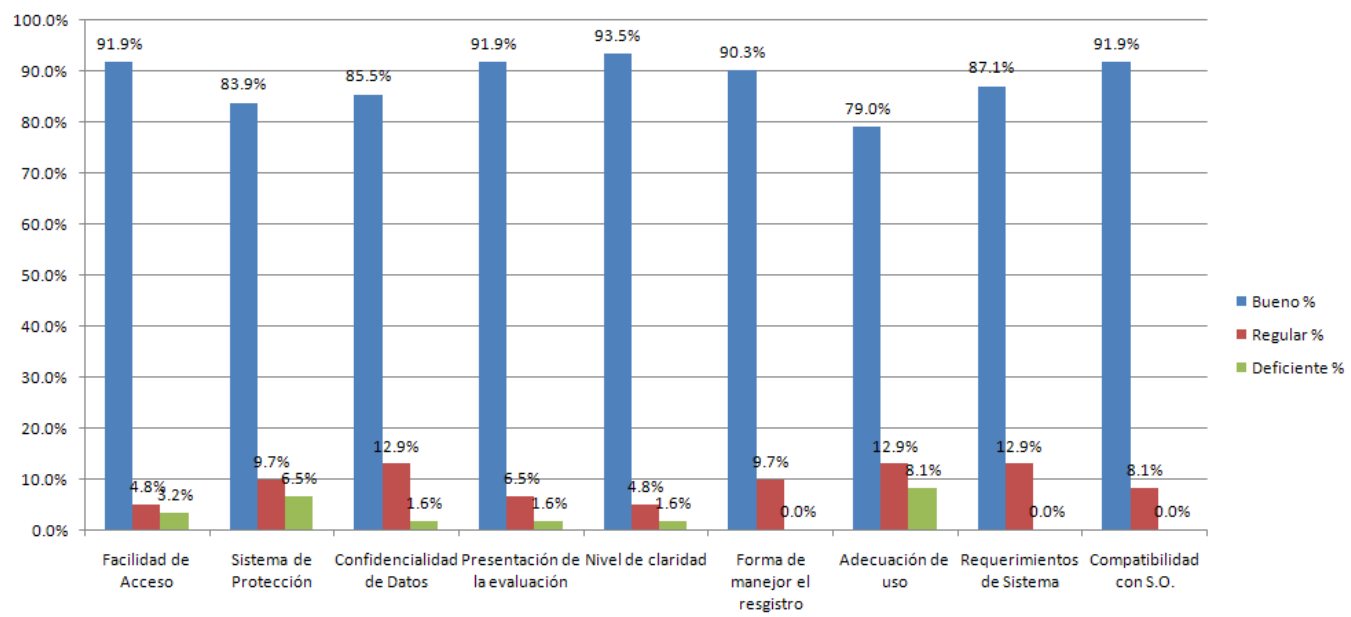

Figura 4: Aplicación del Software de Evaluación de los Aprendizajes para la Obtención de Resultados Académicos

En la Figura 5, se muestran los resultados del estudio de los resultados académicos aplicando el software de evaluación.

$\mathrm{F}=3 \quad\left(\mathrm{n}^{\circ}\right.$ de filas)

$\mathrm{C}=2 \quad\left(\mathrm{n}^{\circ}\right.$ de columnas)

$\mathrm{gl}=2$ (grados de libertad)

$X^{2}{ }_{c}=69,04 ; X^{2} t=9,21 ; g l=2 ; a=0,01$

Donde: $X^{2}{ }_{c}>X^{2}$ por lo que existe diferencia estadísticamente significativa entre el Pre-test y el Post-test; y se puede apreciar que la diferencia entre el pre-test y el post-test es significativamente diferenciada donde la percepción "Bueno", está en el post-test (después de aplicar el software de evaluación) y la percepción de "Regular" y "Deficiente" están en el pre-test (antes de aplicar el software de evaluación). 


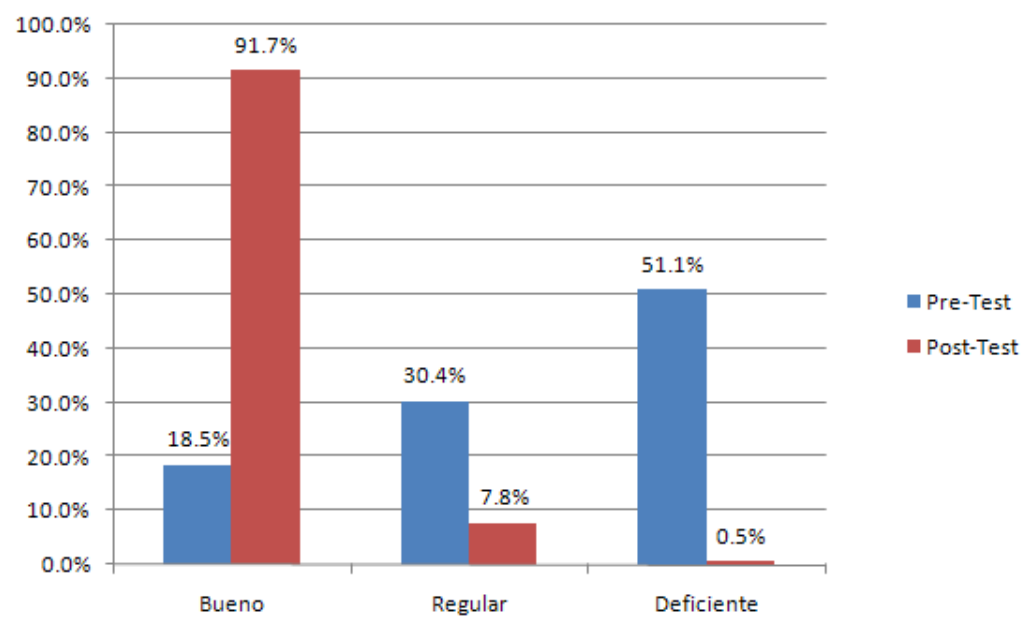

Figura 5: Resultados del estudio de los Resultados Académicos aplicando el Software de Evaluación de los Aprendizajes.

\section{CONCLUSIONES}

- Los estudiantes que conformaron el grupo, antes de la aplicación del software de Evaluación de los Aprendizajes, de Facultad de Industrias Alimentarias de la Universidad Nacional de la Amazonía Peruana en el año 2011 señalan que los resultados académicos de las evaluaciones, es deficiente.

- Los estudiantes que conformaron el grupo, antes de la aplicación del software de Evaluación de los Aprendizajes, de Facultad de Industrias Alimentarias de la Universidad Nacional de la Amazonía Peruana en el año 2011 señalan que si es necesario el uso de un software de Evaluación de los Aprendizajes.

- Los estudiantes que conformaron el grupo, después de la aplicación del software de Evaluación de los Aprendizajes, de Facultad de Industrias Alimentarias de la Universidad Nacional de la Amazonía Peruana en el año 2011 señalan que los resultados académicos de las evaluaciones, son Buenos.

- Los estudiantes que conformaron el grupo, después de la aplicación del software de Evaluación de los Aprendizajes, de Facultad de Industrias Alimentarias de la Universidad Nacional de la Amazonía Peruana en el año 2011 señalan que el uso de un software de Evaluación de los Aprendizajes, da resultados de mejor calidad y en menor tiempo.

- La relación entre aplicación de un software de Evaluación de los Aprendizajes y la Obtención de Resultados Académicos, fue alta en estudiantes de la Facultad de Industrias Alimentarias de la Universidad Nacional de la Amazonía Peruana.

- Existe relación estadísticamente significativa entre la obtención de resultados académicos del grupo antes y después de la aplicación de un software de Evaluación de los Aprendizajes en estudiantes de la Facultad de Industrias Alimentarias de la Universidad Nacional de la Amazonía Peruana en el año 2011.

- El estudio demostró que la aplicación de un software de Evaluación de los Aprendizajes es una herramienta técnico-pedagógica que permite: mejorar el tiempo de desempeño del docente y da mayor satisfacción a los estudiantes de la Facultad de Industrias Alimentarias de la Universidad Nacional de la Amazonía Peruana en el año 2011. 


\section{REFERENCIAS BIBLIOGRAFICAS}

AGUIRRE, J. 2000. Evaluación de Articulación. Compendio. Segunda Edición. Lima - Perú. pp. 6466, 9-11, 89-96, 97-103, 67-72, 511.

ALVAREZ, R. 1997. Computadoras y educación: una propuesta didáctica. Tesis para optar el título de Licenciado en Informática. Universidad Autónoma de Querétaro. México. pp. 5-7, 78-81.

EISER, L.; SALPETER, J. 2001. Introducción a la Informática. Grupo Editorial Iberoamérica, 3ra edición. México. pp. 12-26.

ELLIS, J. 2002. Sistemas Operativos y Compiladores. Mc Graw-Hill, 3ra edición. España. pp. 46-51

HIDALGO, M.2001. Evaluación del Aprendizaje. Edición INADEP. 8a Edición. Lima - Perú. pp. 45, 69.

NORRIS, W. 2005. PC para Principiantes. Computec Editores, 3ra edición. México. (2005). p. 115. pp.74.

ORLICH, D.; et al. 2006. Técnicas de Enseñanza. Modernización en el Aprendizaje. 3ra edición. Editorial LIMUSA S.A. México. pp. 210-215.

ROSI, E. 2002. Evaluación de la Educación. Segunda Edición. Edición E.R. Lima - Perú. pp. 95-96, 96-107, 109-110, 133-135.

Recibido: 06 febrero 2012 / Aceptado: 10 abril 2012 